\title{
Modelling a Single-Blade Wind Turbine using Computational Fluid Dynamics
}

\author{
$\underline{\text { S. J. Wakes }}^{\text {a }}$, G. Gabriel ${ }^{\text {b }}$, P-Y. Potonnier ${ }^{\text {c }}$, A. Dürbaum ${ }^{\text {c }}$, and B. Currie ${ }^{\text {c }}$ \\ ${ }^{a}$ Centre for Materials Science and Technology, University of Otago, Dunedin, New Zealand \\ ${ }^{b}$ Fakultät für Maschinenbau und Schiffstechnik, University of Rostock, Germany \\ ${ }^{c}$ PowerhouseWind, Dunedin, New Zealand \\ Email:sarah.wakes@otago.ac.nz
}

\begin{abstract}
Wind resource does not necessarily occur near areas of high population where the power it could generate can be readily used. It can therefore be uneconomic to site large-scale wind farms in the most suitable areas. New Zealand has a small population that is concentrated in areas that do not necessarily coincide with good wind resource. Transporting power is also a challenge in New Zealand as it is a long thin country with an ageing lines network. An alternative could be distributed household energy generation utilising wind resources at or near point of use (private properties or small community hubs such as schools). Requirements for such wind turbines are likely to differ from those for a commercial wind farm. The scale is reduced with a lower mast, a smaller power output and often a less than ideal site placement due to planning restrictions and other issues such as cost. These factors can lead to a domestic turbine having to utilise highly variable winds to generate power.
\end{abstract}

A unique one-blade downwind stall regulated horizontal axis wind turbine, Thinair 102, has been designed by PowerhouseWind and is in initial production in Dunedin, New Zealand. This turbine is designed for domestic use and to be efficient and effective in such highly variable winds. The turbine is designed to have a high real net output ratio. The turbine is limited to $3 \mathrm{~kW}$ and has a shutoff at $345 \mathrm{rpm}$. The blade is part of the NREL S series and was chosen for its soft stalling capabilities and high angle of attack. The airfoil section has the S835 at the root, S833 as the primary profile and S384 at the tip providing high lift. It has a rotor diameter of $3.6 \mathrm{~m}$ and a rated rotation speed of $370 \mathrm{rpm}$. The freely teetering hub is angled at approximately $7^{\circ}$ (to the y axis) while operating normally. This mechanism allows the blade to change angle in response to variation in the wind speed and allows strong gusty winds to be used efficiently to generate power. In extreme wind conditions the blade swings into a safe horizontal position. The controller for the turbine adjusts the RPM of the blade to maximise the power output although in highly variable winds care has to be taken to avoid rapid changes as this has the potential to put unexpected structural load on the turbine hub. Understanding of the stall behaviour of the turbine therefore becomes important for optimum design of the controller software.

Computational Fluid Dynamics modelling has been used to model the stall behaviour of the turbine blade over a range of wind speeds and RPM in order to better understand the variations in power that can occur in variable winds. Comparison of the CFD simulation results with other commonly used models to calculate wind turbine power output was undertaken. When there was little or no stall on the blade the simpler models for power output gave adequate estimates. However they did not fully account for when stall occurs, giving either higher or lower power output estimates. CFD modeled stall more accurately as well as giving detailed three-dimensional visualisations of the flow over the blade. This allowed examination of the areas of the blade that were contributing to the power output and where stall was occurring over a range of wind speeds and RPM of the blade.

The value of using CFD for a small enterprise to further understand and develop their product was highlighted. By undertaking a specific project with well-defined boundaries PowerHouseWind gained valuable knowledge about their turbine that can be used in the future to further enhance their design and its capability. However it was essential to have a skilled analyst to undertake the work, spend sufficient time understanding the problem and use appropriate models and assumptions to ensure the results were applicable and accurate.

Keywords: $\quad$ Computational Fluid Dynamics, wind turbine, stall behaviour 


\section{INTRODUCTION}

New Zealand's wind resource is considered to be of a high quality (Reuther and Thull, 2011). Some commercial wind farms do take advantage of this resource but there are a number of barriers such as lack of investment and social/environmental concerns (Kelly, 2011). The electricity distribution network in New Zealand requires investment in infrastructure to ensure continuing capacity and security of supply. Distributed supply can be seen to have a number of benefits in New Zealand such as reduced losses and making the supply more geographically diverse (Nair and Zhang, 2009). Requirements for small-scale wind turbines are likely to differ from those for a commercial wind farm. The scale is reduced with a lower mast, a smaller power output and often a less than ideal site placement due to planning restrictions and other issues such as cost. These factors can lead to a domestic turbine having to utilise highly variable winds to generate power.

PowerhouseWind is a company located in Dunedin, New Zealand. The company has developed an innovative one-blade downwind stall regulated horizontal axis small-scale wind turbine (Thinair 102) with a very high real net output ratio that responds to variations in wind speed through the angle of the blade to the wind in order to control and optimise power output. As the wind direction and strength can be constantly shifting this has the potential to affect the output power of the turbine. Nevertheless it is possible to lower the fluctuation of the output power with an optimized design and control system. Aerodynamic stall on turbine blades can lead to less than optimal power output and with variable winds the amount of stall can change rapidly so it is important to understand flow at the turbine and the blade. CFD has the potential to deliver a better understanding of aerodynamic effects such as the stall behaviour and offers the possibility to simulate a big range of different conditions. Most modeling has been done on three blade turbine systems, although some information is available on the effect blade number has on performance of a turbine (Hansen and Madsen, 2011; Hau and von Renouard, 2005; Mo and Lee, 2011). The aim of this work therefore is to model the wind flow over the Thinair 102 blade using Computational Fluid Dynamics. This will enable improved optimization of the turbine controller to enable a more consistent power output at higher wind speeds through better understanding of stall behaviour on the blade.

\section{BACKGROUND}

\subsection{Wind turbines}

Wind turbines extract energy from environmental wind flows. Turbines are characterised as either horizontal axis wind turbines (HAWT) or vertical axis wind turbines (VAWT). HAWTs can use either the physical effect of drag or lift to produce power. Betz law is used to define the maximum power that can be extracted from the wind with the Betz limit for lift-based machines at 59\%. To get close to this Betz limit, using a liftbased HAWT, a knowledge about aerodynamics is required (Ragheb and Rahheb, 2011). Stall is used to regulate power output in high winds with the main requirements for stall-regulated HAWT rotors being:

- maximum performance and efficiency on a big wind speed range $(3 \mathrm{~m} / \mathrm{s}$ to $20 \mathrm{~m} / \mathrm{s})$

- controlled stalling behaviour for wind speeds over rated wind speed

- blade geometry that delivers adequate mechanical properties with economic use of material.

Tip Speed Ratio (TSR) is the ratio between the tangential speed of the tip of the blade and the speed of the wind and is another important consideration for optimal performance of a wind turbine blade. A TSR that is too low does not allow the turbine to extract enough energy from the wind while too high a value creates a large amount of drag. A TSR of 7 is a common value for three blade turbines. If the TSR is too low due to poor blade design there can be a tendency for the blade to slow and stall. Conversely if the TSR is too high the blade will experience larger stresses and therefore could fail in some wind conditions (Ragheb and Rahheb, 2011).

A wind turbine starts to produce power at the cut-in wind speed $v_{c i}$. In the range between $v_{c i}$ and the rated wind speed $\mathrm{v}_{\mathrm{r}}$ the output power increases as the cube of the wind speed, because the output power $\mathrm{P}_{\text {out }}$ is related to the wind speed $\mathrm{v}_{\mathrm{w}}$ (Hau and von Renouard, 2005).

$$
P_{\text {out }} \propto v_{w}^{3}
$$

At wind speeds between $\mathrm{v}_{\mathrm{r}}$ and the cut-out wind speed $\mathrm{v}_{\mathrm{co}}$ the rotor uses stall regulation to retain the output at the rated output $P_{\text {rated. }}$. Without this regulation loads would damage the turbine.

The intensity of the stall effect depends on the angle of attack of the aerodynamic profile. The angle of attack is connected to the wind speed and the RPM of the rotor. As the angular velocity of the blade is adjusted by 
the turbine control system the intensity of the stalling effect changes. Power output, stall, ratio of lift coefficient $C_{L}$ to drag coefficient $C_{D}$ and angle of attack are related. The greater the effect of stall, the lower the output power and the lower the ratio of $C_{L}$ to $C_{D}$. Small changes in the angle of attack can have a significant effect on the ratio of $C_{L}$ to $C_{D}$ in the region where stall occurs.

\subsection{ThinAir 102 Wind Turbine}

The ThinAir 102 wind turbine operates downwind and has a rated output of $2 \mathrm{~kW}$. The wind turbine has a patented teetering hub, which allows the blade to teeter out of the rotor plane into a safe horizontal flag position (Figure 1). This happens on heavy wind or wind gusts and minimizes the swept area of the blade. This function allows the turbine to operate in heavy winds without getting damaged (PowerhouseWind, 2013). The freely teetering hub is angled at approximately $7^{\circ}$ (to the horizontal) while operating normally. This mechanism allows the blade to change angle in response to variation in the wind speed and allows strong gusty winds to be used efficiently to generate power. The blade is balanced using two counterweights (each $5 \mathrm{~kg}$ and $0.36 \mathrm{~m}$ long). The controller for the turbine adjusts the RPM of the blade to maximise the power output although in highly variable winds care has to be taken to avoid rapid changes as this has the potential to put unexpected structural loads on the turbine hub. The airfoil profile used is part of the S-Series offered by NREL and is specially designed for wind turbines. For wind turbine rotors with a diameter between one and three metre NREL recommends the S835 for the root, S833 at primary and the S834 for the tip with all three profiles providing a high lift coefficient (Hansen and Madsen, 2011).

The Thinair 102 wind turbine is soft-stall regulated with variable rotation speed rotor that uses an electrical brake. By gradually slowing the blade as the wind increases, the blade then progressively stalls, sheds its aerodynamic power and regulates the power output. The amount of brake applied on the rotor is automatically computed by control software designed by PowerhouseWind using an iterative control process on the RPM and power output values. Although the software is efficient and effective in most cases, one of its limits is dealing with strong, almost instantaneous gusts of wind.

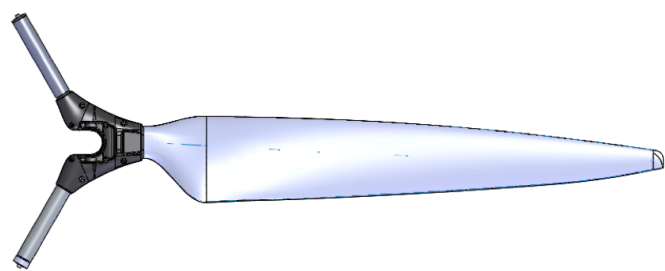

(a)

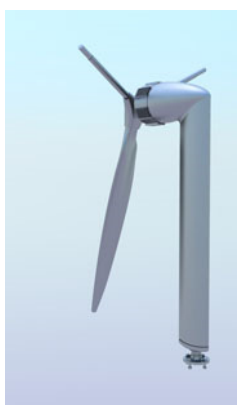

(b)

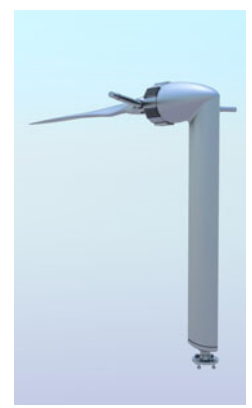

(c)

Figure 1. Thinair 102 wind turbine (a) blade with counter balance weights and teetering hub, (b) in normal operating mode and (c) blade fully teetering in extreme winds (PowerhouseWind, 2013).

\section{SIMULATIONS}

\subsection{Computational Fluid Dynamics Model}

The NREL Unsteady Aerodynamics Experiment, where a real two bladed rotor with a 10m diameter was tested in NASA AMES wind tunnel allowed a full-scale turbine to be tested in a controlled environment with a minimum of turbulence. This is now a test for CFD research communities to verify the reliability of different CFD codes. It was found that Navier-Stokes based codes can deliver results comparable to the experimental results (Hansen and Madsen, 2011).

The rotor coordinate system has its origin at the rotor centre, rotates with the rotor and turns with the azimuth of the turbine. The $\mathrm{x}$-axis points in direction of the rotor axis and which in this case is also downwind. The $\mathrm{z}-$ axis points towards the blade tip and the y-axis is perpendicular in accordance with the right hand rule. While operating the rotor rotates clockwise around the x-axis. For this work the geometry of the blade is the ThinAir 102.

The domain size for this project is based on a CFD investigation of a small-size wind turbine of the NREL PHASE VI project (Hansen and Madsen, 2011) using ANSYS Fluent. The domain is a cylinder with a 
diameter and length six times the rotor radius. The cylindrical domain is centred on the origin of the rotor coordinates and is two rotor radii in front of the rotor. With a rotor radius of $1.8 \mathrm{~m}$ the domain is $10.8 \mathrm{~m}$ long and has a diameter of $10.8 \mathrm{~m}$.

Air with a density of $1.225 \mathrm{~kg} / \mathrm{m}^{3}$ and a viscosity of $1.7894 \times 10^{-5} \mathrm{~kg} / \mathrm{m} / \mathrm{s}$ is used in the simulations. Turbulence is modeled using the shear-stress transport (SST) model, a well understood model that offers a good compromise between accuracy and computational effort (Alexandrou, 2001). The reference frame rotates around the $\mathrm{x}$-axis of the rotor coordinate system at angular velocities between 297 and $345 \mathrm{rpm}$. The surface of the blade is set as stationary and the boundary is no-slip. Wind speeds are between 8 and $18 \mathrm{~m} / \mathrm{s}$ and cover the whole range at which the turbine is operating and where stalling could occur. Conversation of mass is attained to within $0.001 \%$. A mesh density study was undertaken with a final mesh of 9.4 million elements used that balances sufficient numerical accuracy with an acceptable simulation time of around 24 hours per case for a 30GByte RAM computer.

The torque $\mathrm{M}$ of the blade to the rotor axis is an output from the simulations and used to calculate the produced power of the blade with the angular velocity of the blade $\omega$.

$$
P_{\text {out }}=M \omega
$$

\subsection{Other models and $\log$ files}

Two two-dimensional simulation methods are used for comparison purposes in this work. PropID is based on the Blade Element Momentum method (BEM) and includes a three-dimensional post-stall airfoil performance synthesization method for better power prediction at high wind speed (UIUC Applied Aerodynamic Group, 2016). FAST uses the Beddoes-Leishman dynamic stall model for HAWT (NREL, 2016). These two-dimensional models fail to consider any aerodynamic effects that would be related to the rotation of the flow and design problems, e.g. peak power and load fatigue. PropID does allow for correction of tip losses, hub losses, skewed wakes and stall delay.

Validation log files produced by the monitoring system are used to get data from an operating wind turbine. These $\log$ files save wind speed, RPM and output power of the alternator every five milliseconds. This data was scanned and arranged to be comparable to cases. Results for each case were averaged and the standard deviation was also considered. The losses in power between the turbine and that recorded at the alternator range between 9 to $13 \%$ and are RPM dependent. For some cases the data was not usable or does not exist.

\section{RESULTS}

For cases with TSR close to the optimum 7 the flow should be mostly attached. At $9 \mathrm{~m} / \mathrm{s}$ with $321 \mathrm{rpm}$ and $345 \mathrm{rpm}$ (TSR 6.72 and 7.23) the results show very good agreement with this assumption (Figure 2a). At 13 $\mathrm{m} / \mathrm{s}$ stall at the tip of blade occurs (Figure 3). Greater deviations between the different approaches are also seen as wind speed increases. Nevertheless all approaches show the same trend over the different cases and keep the same order (Figure 2). PropID predicts the lowest power; FAST the highest output power, with the CFD results located in-between. If the losses between the power produced by the turbine and that recorded at the alternator in the log files ( 9 to $13 \%$ ) are accounted for the CFD results give the closest predictions.

At $15.5 \mathrm{~m} / \mathrm{s}$ and $18 \mathrm{~m} / \mathrm{s}$ stall regions are starting to dominate (Figure 2c, $d$ and 3). For the cases with a lower TSR, differences of up to $100 \%$ in power predicted occur between PropID and FAST (Figure 2d). The CFD results are still located in-between these. FAST only allows two or three bladed wind turbines as an input. For these calculations the three-bladed setup was used with the second and third blade in the setup as the cylindrical geometry of the two counterweights, which potentially results in additional power generation prediction from these structures, affecting the high final results. Secondly, the software may not be suited for such small wind turbines, which perform at very high rotational speed. High rotational speeds have a strong influence on the three dimensional effects of the flow, especially at stall. At TSR lower than 3.76 the PropID and CFD predictions show very good agreement with each other (Figures $2 \mathrm{c}$ and $2 \mathrm{~d}$ ).

The angle of attack (AoA) has the biggest influence on the stalling behaviour in these cases and it is closely related to the TSR. Decreasing TSR leads to higher AoA. A TSR of 7 is the optimum tip speed ratio of the Thinair 102. Around TSR 7 the flow is completely attached and delivers the highest efficiency or power coefficient $\mathrm{C}_{\mathrm{p}}$ (Figure 3). At TSR 5 the first stall appears at the tip and this region increases in size until TSR 4.31. The tip stall region between radius value $1.4 \mathrm{~m}$ and $1.8 \mathrm{~m}$ remains a similar size until the separated flow is over the whole suction side of the blade. A second stall region occurs at the same time over the whole trailing edge that grows with declining TSR values (Figure 3). 
The third stall area appears around TSR 4.31 at a radius of $1.2 \mathrm{~m}$. The development of this region is rapid between TSR 4.20 and TSR 3.83 and the power coefficient decreases by 20 to $30 \%$ in this TSR range. This region continues to grow, but not that rapidly as TSR decreases. The region spans radius $0.8 \mathrm{~m}$ to $1.4 \mathrm{~m}$ at TSR 3.76 (Figure 3). For TSR 3.61 and lower it is difficult to differentiate the different stall regions as separated flow occurs all over the suction side of the blade (Figure 3). The power coefficient is very low and aerodynamic effects are hard to predict. The wind turbine shuts down at wind speeds higher than $18 \mathrm{~m} / \mathrm{s}$.

The development and effect of the tip stall between radius $1.4 \mathrm{~m}$ and $1.8 \mathrm{~m}$ at a wind speed of $13 \mathrm{~m} / \mathrm{s}$ shows that regions with separated flow produce less torque than regions of attached flow. Additionally the size of the areas of separated flow is strongly related to the amount of loss of torque (Figure 4a). For a wind speed of $15.5 \mathrm{~m} / \mathrm{s}$ the flow behaves the same for all four cases in the tip region and this is reflected in the similar power output generated in the region of the blade between 1.4 and $1.8 \mathrm{~m}$. At $345 \mathrm{rpm}$ a small area of separated flow occurs at $1.2 \mathrm{~m}$ along the blade, which then grows as TSR decreases. The predicted torque decreases as a consequence of the stall region in the centre of the blade growing (Figure $4 \mathrm{~b}$ ).

(a)
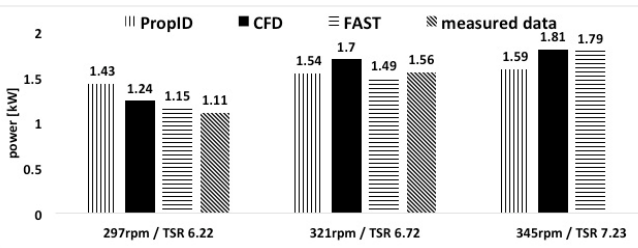

(b)
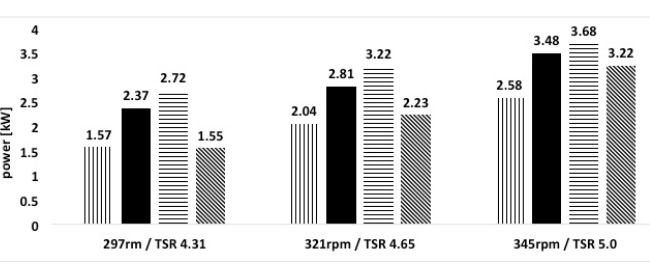

(c)
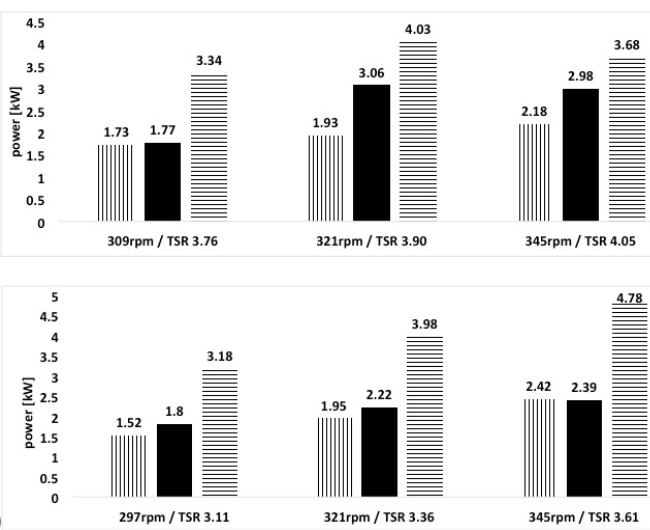

(d)

Figure 2. Power predictions for CFD, PropID, FAST simulations and $\log$ files for (a) $9 \mathrm{~m} / \mathrm{s}$, (b) $13 \mathrm{~m} / \mathrm{s}$, (c)

$15.5 \mathrm{~m} / \mathrm{s}$ and (d) $18 \mathrm{~m} / \mathrm{s}$ for varying blade RPM

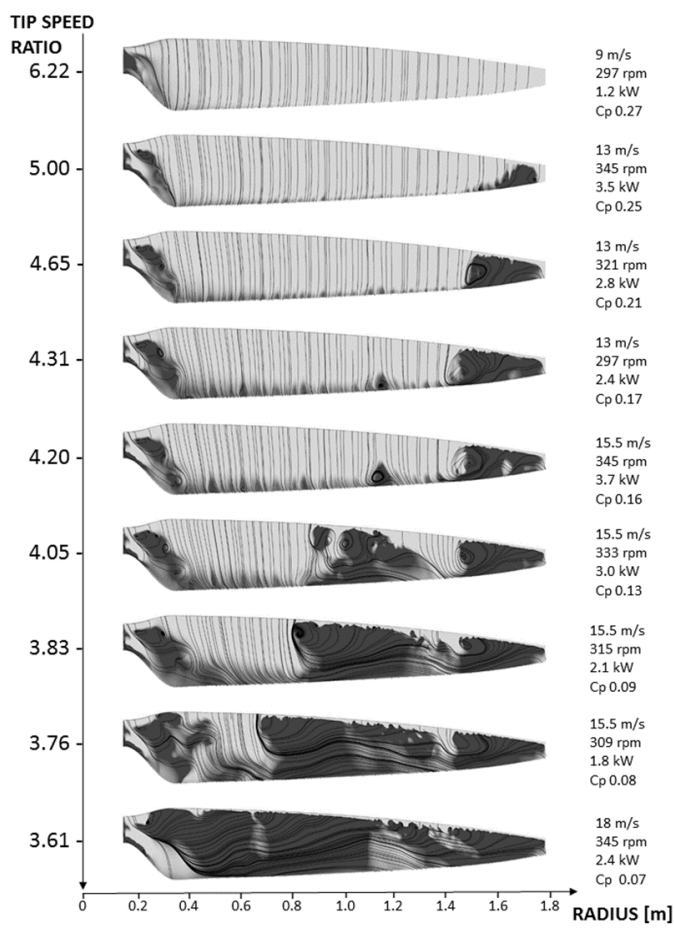

Figure 3. Stall visualisation on the Thinair blade with varying TSR. Dark grey areas represent separated flow and light grey areas attached flow with the lines showing the surface streamlines.

\section{DISCUSSION AND CONCLUSIONS}

In the region of attached flow the three simulation models and the log files show good agreement. As soon as stall occurred the modeling approaches showed different behaviour. PropID, with the lowest values for the power output, may predict stall very early and at lower TSR while FAST predicts attached flow even in cases of low TSR. At TSR 5 CFD predicts mostly attached flow and as high an output power as the FAST calculations. Between TSR 5 and 3.76 there is a transition in the CFD results, where it goes from high power output predictions like the FAST model to lower predictions as from the PropID model. At very low TSR the CFD code shows very good agreement with results from PropID, but there are no log files for validation. Verification and validation show that the CFD code is always in the same range as these other specialized models used for HAWT and there is good agreement with the log files. Furthermore all predictions display 
the same trends over all cases. The biggest deviations occur in the region of transition between attached and separated flows on the blade. This is probably the most difficult region in which to predict flow behaviour, but it is also the region of most interest. A significant transition in stall of the blade is predicted by the CFD simulations in the region between TSR 3.5 and TSR 5.

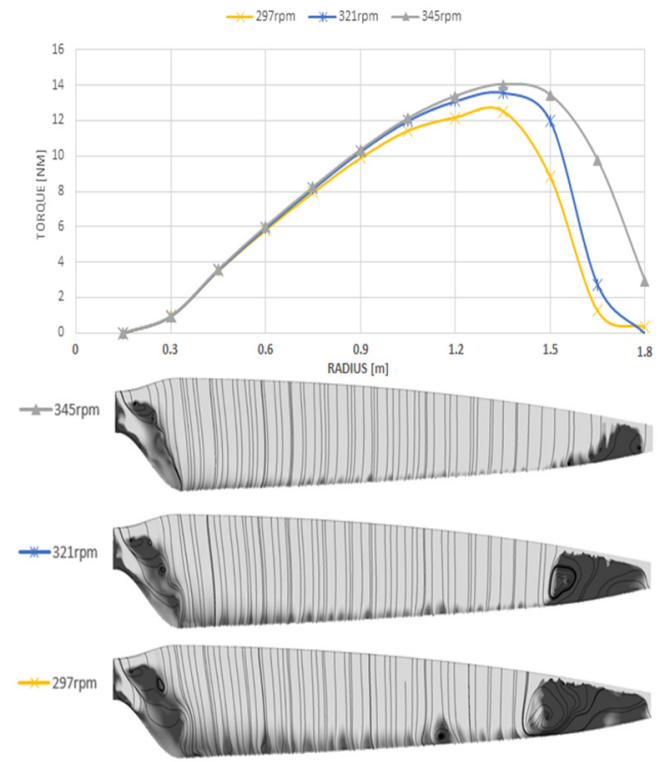

(a)

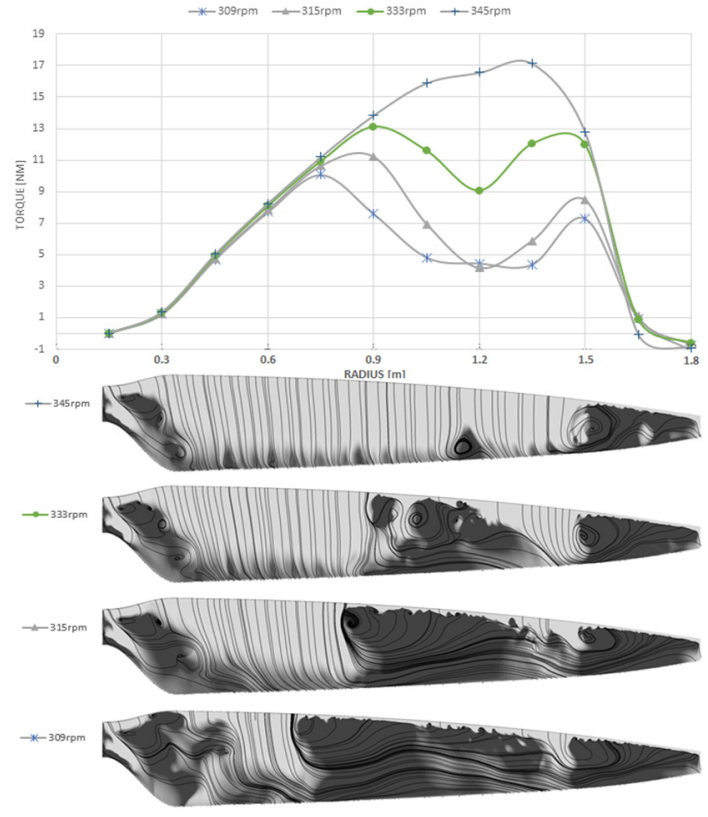

(b)

Figure 4. Torque distribution and stall visualization for wind speeds (a) $13 \mathrm{~m} / \mathrm{s}$ (b) $15.5 \mathrm{~m} / \mathrm{s}$ at varying RPM

The results show that there are three different areas on the suction side of the blade with different stalling behaviour. The first area is located at the tip of the blade between radius $1.4 \mathrm{~m}$ and $1.8 \mathrm{~m}$. Although this section has the longest lever arm the torque drops rapidly in this region and the area on the blade is relatively small so the influence on the power output is small. The small change is related to wind speed or RPM changes and happens continuously. This reduces the power at higher wind speeds, which is the main aim of the stall regulation, and as the changes are continuous does not interfere with the control system. The second area is on the trailing edge with stalling that grows consistently with decreasing TSR and produces a very smooth regulation of the output power, beneficial for the control system.

The third area, which produces the most power, is located between $0.8 \mathrm{~m}$ and $1.4 \mathrm{~m}$ on the blade. Between TSR 3.83 and TSR 4.20 this area has a significant change in efficiency and produced power. With fluctuating wind speeds large abrupt changes could occur producing strongly fluctuating output power. In the long-term such fluctuations can damage the turbine converter. This is demonstrated by the following example: the turbine running at $345 \mathrm{rpm}$ in a wind speed of $18 \mathrm{~m} / \mathrm{s}$ gets close to maximum power of $2.5 \mathrm{~kW}$, with stall occurring due to flow separation. Over a short time the wind could easily drop to $15.5 \mathrm{~m} / \mathrm{s}$ with the flow then mostly reattaching while the turbine is still running at $345 \mathrm{rpm}$. The turbine power output will then be 3.5 to $4 \mathrm{~kW}$, over the limiting values of the alternator, and potentially resulting in damaged or destroyed components.

These abrupt changes can be explained in part by three-dimensional effects, which occur on the rotating blades due to vortex generation at the blade root and tip and the radial forces. The flow is therefore attached for longer at higher wind speeds compared to non-rotating blades. Additionally when one part of the flow is attached but the flow next to it is going to separate, the attached flow interacts with the separated flow, which wants to peel off, and keeps it attached. Then at the point of transition where the flow is not able to stay attached the whole area peels off at once. This could be a reason for the high fluctuating power output of the Thinair 102 around TSR 4. (Hi et al., 2006; Mo and Lee, 2011; Pape and Lecanu, 2004).

By using CFD to simulate the Thinair 102 wind turbine understanding has increased for PowerhouseWind about their product in a variety of wind and use scenarios. The detailed knowledge of stall behaviour enables identification and verification of potential problem areas and their causes, and may drive further design enhancements to the system in the future. For a small business with no ongoing ability to undertake such time 
consuming and complex simulations a discrete simulation project with clearly defined boundaries has been beneficial. It was essential that the ongoing dialogue with the business was maintained to keep the project on track and to deliver useable and useful results. Even for a skilled analyst time was needed to understand the problem, become familiar with relevant research and make appropriate decisions regarding models and assumptions used (Bezerra and Wakes, 2005; Fahey and Wakes, 2005; Wakes et al., 2010). CFD simulations produced detailed three-dimensional data sets that can be used in a number of ways and predicted valid power output values that are comparable to actual turbine data. However the simpler two-dimensional models were shown to be appropriate to use to predict power output in some circumstances. These have the advantage over CFD models of shorter lead and simulation times, are less resource intense, but limited in their ability to predict stall and its subsequent effect on power output.

PowerhouseWind are now able to make an informed decision about which of these models to use going forward to predict power output. This project demonstrated that it is possible for a small business to use a challenging analysis tool such as CFD to aid understanding of a complex physical environment, as well as the more usual uses such as troubleshooting design issues and technical problem solving (Bezerra and Wakes, 2005). An additional bonus is that simulation data can be used to generate materials for further investment bids and marketing by demonstrating the viability of the technology.

\section{REFERENCES}

Alexandrou AN (2001). Principles of Fluid Mechanics, Prentice-Hall.

Bezerra C and Wakes S (2005). Designing intellegence and the role of computer-supported methods for innovation. In: Proceedings of 6th International conference on computer-aided industrial design \& conceptual design, Delft, Netherlands, pp. 362-367.

Fahey M and Wakes S (2005). Assisting product development with Computational Fluid Dynamics. In: Proceedings of 6th International conference on Computer-aided Industrial Design \& Conceptual Design, Delft, Netherlands, pp. 629-635.

Hansen MOL and Madsen HA (2011). Review paper on wind turbine aerodynamics. Jounal of Fluid Engineering, 133(11): 1-12.

Hau E and von Renouard H (2005). Wind turbines: Fundamentals, Technologies, Application, Economics, Springer.

Hi D, Hua O and Du Z (2006). A study on stall-delay for horizontal axis wind turbine. Renewable Energy, 31: 821-836.

Kelly G (2011). History and potential of renewable energy development in New Zealand. Renewable and Sustainable Energy Reviews, 15(5): 2501-2509.

Mo J-O and Lee Y-H (2011). CFD Investigation on the aerodynamic charateristics of a small-sized wind turbine of NREL PHASE VI operating with a stall-regulated method. Journal of Mechanical Science and Technology, 26: 81-92.

Nair N-KC and Zhang L (2009). SmartGrid: Future networks for New Zealand power systems incorporating distributed generation Energy Policy, 37: 3418-3427.

NREL (2016). FAST. Retrieved from https://nwtc.nrel.gov/FAST

Pape AL and Lecanu J (2004). 3D Navier-Stokes computations of a stall-reglated wind turbine. Wind Energy, 7: 309-324.

PowerhouseWind (2013). Thinair specification. $\quad$ Retrieved 6/7/17, from http://www.powerhousewind.co.nz/products.htm.

Ragheb M and Rahheb AM (2011). Wind turbines theory - The Betz equation and optimal rotor tip speed ratio. In: R C (ed), Fundamental and Advanced Topics in Wind Power (pp. 19-38). INTECH.

Reuther N and Thull J-P (2011). Feasibility Study of Small and Micro Turbines for Residential Use in New Zealand, Land Environment and People Research.

Wakes SJ, Fahey M and Shaw CT (2010). Enhancing and optimising the use of engineering tools within the design process. Design Principles \& Practices: An International Journal, 4(1): 23-32.

UIUC Applied Aerodynamic Group. (2016). PropID. from http://m-selig.ae.illinois.edu/propid.html. 